\title{
The effect of fibre dispersion on initial failure strain and cluster development in unidirectional carbon/glass hybrid composites
}

\author{
Yentl Swolfs ${ }^{* 1}$, Robert M. McMeeking ${ }^{2,3,4}$, Ignaas Verpoest ${ }^{1}$, Larissa Gorbatikh ${ }^{1}$ \\ ${ }^{1}$ Department of Materials Engineering, KU Leuven, Kasteelpark Arenberg 44 bus 2450, 3001 Leuven, Belgium \\ ${ }^{2}$ Department of Mechanical Engineering, University of California, Santa Barbara, CA 93106, USA \\ ${ }^{3}$ Materials Department, University of California, Santa Barbara, CA 93106, USA \\ ${ }^{4}$ School of Engineering, University of Aberdeen, King's College, Aberdeen AB24 3UE, Scotland, UK \\ *Corresponding author: Y. Swolfs (yentl.swolfs@mtm.kuleuven.be), Tel.: +32 16.37.36.16
}

\begin{abstract}
By adding glass fibres to carbon fibre composites, the apparent failure strain of the carbon fibres can be increased. A strength model for unidirectional hybrid composites was developed under very local load sharing assumptions to study this hybrid effect. Firstly, it was shown that adding more glass fibres leads to higher hybrid effects. The hybrid effect was up to $32 \%$ for a hybrid composite with a 10/90 ratio of carbon/glass fibres. The development of clusters of broken fibres helped to explain differences in the performance of these hybrid composites. For 50/50 carbon/glass hybrids, a fine bundleby-bundle dispersion led to a slightly smaller hybrid effect than for randomly dispersed hybrids. The highest hybrid effect for a 50/50 ratio, however, was $16 \%$ and was achieved in a composite with alternating single fibre layers. The results demonstrate that thin ply hybrids may have more potential for improved mechanical properties than comingled hybrids.
\end{abstract}

Keywords: A. Hybrid composites; A. Carbon fibres; B. Synergism; C. Modelling

\section{Introduction}

Carbon fibre-reinforced composites display excellent mechanical properties and a low density, but they often lack toughness and have a limited failure strain. Many strategies exist for improving composite toughness. Adding rubber inclusions [1] or nanoreinforcements $[2,3]$ to the matrix can improve matrix-dominated properties, but these strategies unable to significantly improve the failure strain of carbon fibre-reinforced composites. Ductile fibres, such as metal [4] or polymer fibres [5], can yield high failure strains, but sacrifice either density, stiffness or strength.

One strategy that has potential to combine high stiffness and strength with low density, is fibre hybridisation. This means that two types of fibres are combined to form a hybrid composite [6-9]. The most common hybridisation fibre for carbon fibre composites is glass fibre. In addition to their good mechanical properties, glass fibres are cheaper and thus can lead to significant cost reduction compared to an all-carbon fibre composite. Hybrid composites also possess an interesting balance in mechanical properties. Most properties have mechanical properties intermediate to the two composites consisting of only one fibre type.

One remarkable effect that can be achieved in carbon/glass fibre hybrids is the apparent failure strain enhancement of the carbon fibres. This effect, referred to as the hybrid effect, was first described by Hayashi [10]. By adding glass fibre layers, Hayashi 
succeeded in increasing the failure strain of the carbon fibre layers by $40 \%$. Several authors obtained positive hybrid effects of up to $50 \%$ by this measure [11-15]. It has been reported that the hybrid effect is larger in composites with lower carbon fibre content [8] and better fibre dispersion $[11,16,17]$.

Three hypotheses have been coined to explain the hybrid effect in unidirectional composites: (1) changes in the sequences and mechanisms by which failure develops, (2) the influence of thermal residual stresses on failure, and (3) the consequences of dynamic effects $[13,18]$. The focus here will be on the first hypothesis, as the other two are generally considered to be secondary $[8,17]$. Failure in non-hybrid composites starts when the weakest fibre breaks, thereby losing its local load transfer capability. The load transfer is taken up by the matrix that re-distributes load to surrounding fibres through shear stresses. Away from the break, the axial stress in the broken fibre is recovered to the nominal level. The part of the fibre where this stress is not yet recovered is known as the ineffective length. As a result, the nearby fibres are subjected to local stress concentrations, increasing their failure probability. Therefore, the composite has a tendency to develop clusters of broken fibres, which then lead to even larger stress concentrations in neighbouring intact fibres. At a certain point, the break-cluster reaches a critical size after which it behaves like a large defect that propagates. This rapidly leads to final composite failure.

If a second fibre type is added, the failure development can be significantly altered [19, 20]. No direct observations have been reported, but the following changes can be expected. Firstly, the stress concentrations and ineffective length in each fibre depend on its own fibre type and the type of its neighbours [15, 21, 22]. Secondly, when a break-cluster of low elongation fibres is formed, it can be bridged by unbroken fibres of the high elongation type [23-25]. This can delay further development of the breakcluster and require a larger critical cluster size prior to final failure of the composite. Thirdly, size scaling effects can occur. For a constant sample size, the total number of carbon fibres decreases by partially replacing carbon by glass fibres. Just from this effect alone, the failure strain of carbon fibre layers is expected to be higher in the hybrid composite [26-28].

Over the years, the hybrid effect has been investigated numerically. Some of the older models use 1D packings, composed of a single row of alternating carbon and glass fibres [15, 21, 29]. These packings always contain the same number of carbon and glass fibres. Furthermore, these packings have a fixed fibre dispersion, meaning a carbon fibre is always surrounded by two glass fibres. The models are thus unable to investigate the influence of hybrid volume fraction and fibre dispersion. Furthermore, the failure development in a single row of fibres is not representative of a real hybrid composite. Recently, Mishnaevsky and Dai [30,31] developed a more advanced model for predicting the failure strain of hybrid composites. Using 2D packings, these authors demonstrated the importance of dispersion for damage development, but did not investigate the hybrid effect.

There is plenty of experimental evidence that the fibre dispersion and the relative fraction of both fibres are crucial for maximising the hybrid effect [8]. The mechanisms by which these factors influence failure development in hybrid composites, however, 
remain unclear. The present paper aims to reveal these mechanisms of failure development through modelling.

\section{Strength model for a hybrid composite}

\subsection{General approach}

The model focuses on changes in the sequences and mechanisms by which failure develops. The influence of dynamic effects and thermal residual stresses are not considered in the model. The scheme of the model is summarised in the flow chart in Fig. 1. Based on the parameters described in "2.2 Model parameters", a representative volume element (RVE) is created.

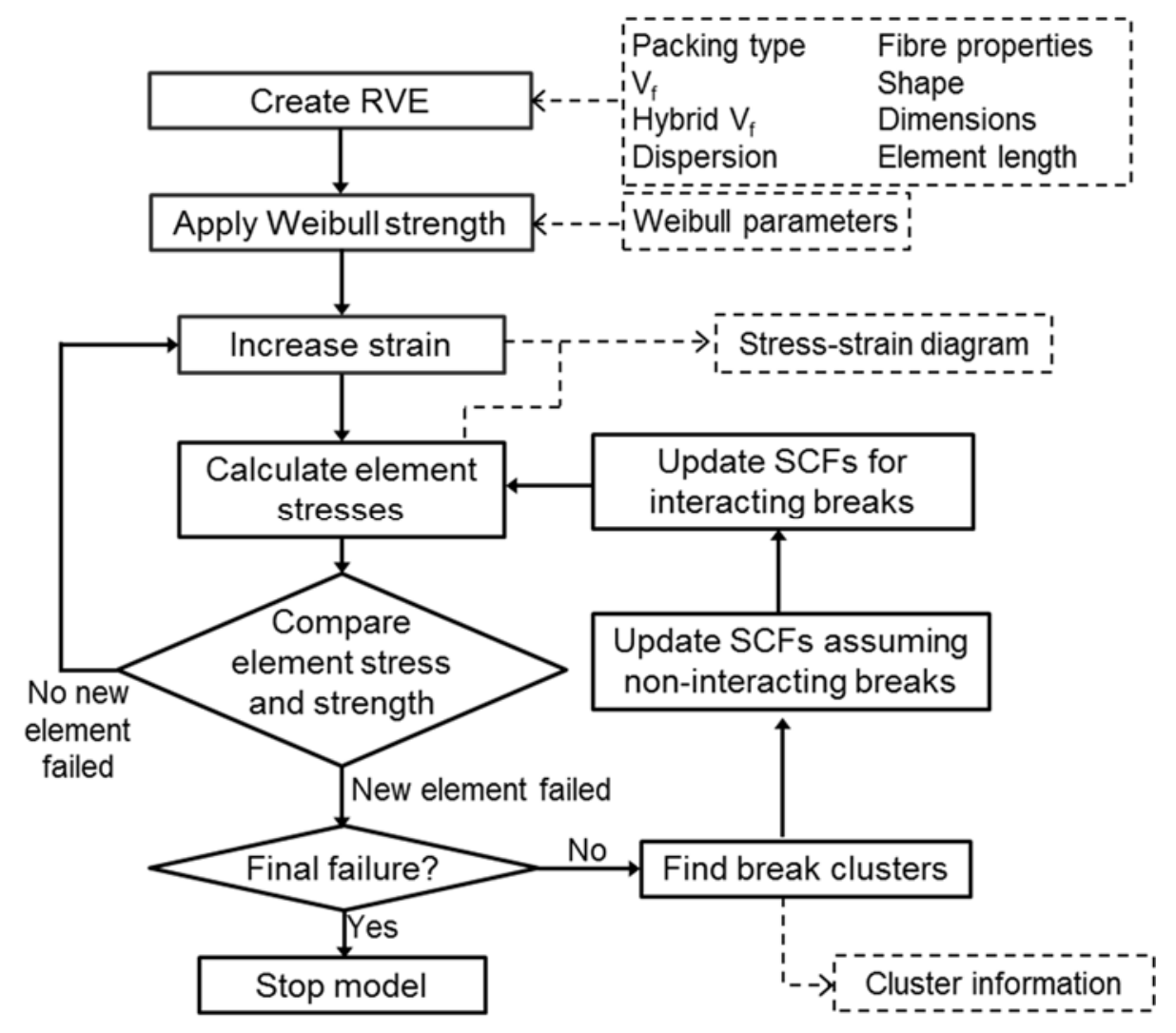

Figure 1: Flowchart for the steps in the procedure for simulating failure of fibre reinforced composite materials. The dashed rectangles indicate inputs and outputs.

The model utilises the chain-of-bundles approach of Rosen [32]. As illustrated in Fig. 2, this approach divides each fibre longitudinally into elements. In the beginning of each Monte Carlo simulation, a strength value consistent with a modified Weibull distribution is assigned to each fibre element. The global strain in the model is gradually incremented. Next, the stress in each fibre element is calculated. The stress $\sigma_{i}$ in element ' $\mathrm{i}$ ' is calculated as $\sigma_{i}=\varepsilon \cdot E_{i} \cdot S C F_{i}$, where $\varepsilon$ is the global strain, $E_{i}$ is the Young's modulus of the fibre, and $S C F_{i}$ is the stress concentration factor applied to 
element ' $\mathrm{i}$ '. The definition and updating procedure for these SCFs will be clarified in "2.3 Stress concentrations without fibre break interactions" and "2.4 Stress concentrations with fibre break interactions".

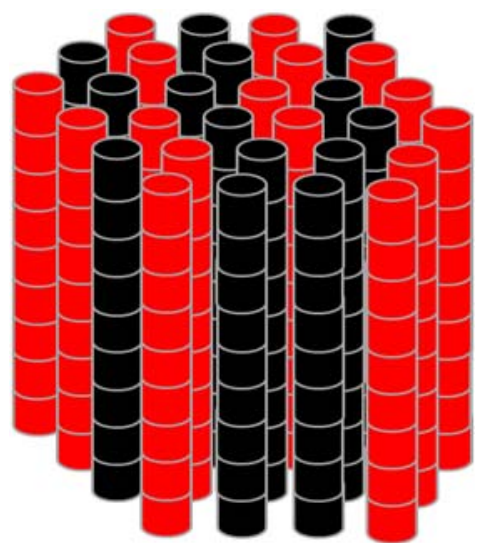

Figure 2: Illustration of how the chain-of-bundles approach splits up fibres into fibre elements.

The model then checks whether any of the fibre elements is carrying a stress higher than its strength. If no new elements are broken, then the strain is further incremented. If new elements are broken, however, the model checks the criterion for final failure. The model is interrupted if more than $10 \%$ of the fibres are broken within an axial segment having a length equal to $35 \mu \mathrm{m}$ or 10 fibre elements. This failure criterion was chosen because its satisfaction always coincides with an exponential increase in the number of fibre breaks. The number of breaks and break cluster size starts to run away at this stage, indicating the propagation of the critical cluster. Further computation of SCFs and fibre breaks becomes both slow and pointless as all fibres will quickly break thereafter.

If the failure criterion is not yet satisfied, then the model searches for the break-clusters. The break-clusters will be defined in " 2.5 Break-cluster development". Updating the break clusters is required for calculating the SCFs around interacting breaks later in the model. Before calculating these SCFs, however, the SCFs around the newly broken fibres are computed assuming that there are no interactions between fibre-breaks. Then, these SCFs are modified to account for possible interactions between fibre breaks. Both these steps will be explained in detail in " 2.3 Stress concentrations without fibre break interactions" and "2.4 Stress concentrations with fibre break interactions".

Since the stress on the neighbouring fibres will increase due to the SCFs, the element stresses in the nearby fibres are calculated again. The process of updating element stresses, checking element failure and updating SCFs is repeated until no new elements fail in the same strain increment. The model then increases the strain and repeats the procedure until the composite failure criterion is satisfied. The strain increment is initially set to $0.04 \%$, but is gradually decreased as the simulation progresses. Near final failure of the composite, the strain increment is reduced to $0.0025 \%$. This gradual refinement results in an optimal combination of computational efficiency and accuracy near final failure. 
The model output is generated by extracting the composite stress as well as the breakcluster information at each strain increment. The composite stress is calculated by weighting the average fibre and matrix stresses by their relative volume fractions. The average fibre stress is obtained from averaging all the element stresses. The average matrix stress is found by multiplying the global strain by the tensile modulus of the matrix.

The hybrid effect is calculated as the relative increase of the failure strain of the hybrid composite compared to the failure strain of the all-carbon fibre-reinforced composite. It should be noted that failure of the hybrid composite in the present model coincides with failure of the carbon fibres. The calculation of the hybrid effect therefore corresponds to the correct definition of the hybrid effect, as recently identified in the review paper by Swolfs et al. [33]. Final failure of the hybrid composite may involve debonding or delamination of glass fibre layers or bundles, but these features are not included in the present model.

Carbon fibre fragmentation or multiple fractures of the carbon fibre layers or bundles $[19,20]$ were not possible in the present model. The model is interrupted when the first critical cluster propagates. As the model does not predict what happens to the glass fibres after this has occurred, fragmentation cannot be captured in the present model. This also causes the predicted stress-strain diagrams to be linear.

\subsection{Model parameters}

The model uses hexagonal fibre packings, as this facilitates the prediction of stress redistribution among broken and unbroken fibres. The choice for hexagonal packings over more realistic random packings [34] is also motivated by the usage of the very local load sharing rule. As will become clear in "2.3 Stress concentrations without fibre break interactions", this rule is more straightforward to apply in hexagonal packings. Square packings are also possible, but they would lead to higher and less realistic stress concentrations.

The difference in diameter between carbon and glass fibres restricts the maximal fibre volume fraction in a hexagonal packing with both these fibres. To avoid this limitation, both fibres are assumed to have the same diameter, allowing for an overall fibre volume fraction of $50 \%$. Fibre types are assigned to individual fibres through various schemes so that the influence of dispersion and hybrid volume fraction can be investigated. The hybrid volume fraction is defined as the ratio of volume of glass fibres over the overall fibre volume.

The fibre radius is chosen to be $3.5 \mu \mathrm{m}$ for both carbon and glass fibres, and the elastic moduli of the carbon and glass fibres are 230 and $70 \mathrm{GPa}$, respectively. The tensile modulus of the matrix is $3 \mathrm{GPa}$.

Each fibre is divided into 2857 fibre elements of $3.5 \mu \mathrm{m}$ length, and thus the total gauge length is $10 \mathrm{~mm}$. The cylindrical model contains 2276 fibres, including 276 boundary fibres around the model perimeter. The reason for these boundary fibres will be explained in "2.4 Stress concentrations with fibre break interactions". 
A total of 50 realisations is performed for each type of hybrid composite. Each realisation has a different Weibull strength assignment for the fibre elements. The error bars on all figures indicate the $95 \%$ confidence intervals.

Several researchers have pointed out that extrapolation of the standard Weibull distribution down to short gauge lengths leads to overestimation of carbon fibre strength [27, 35-37]. Authors have therefore proposed a modified Weibull strength distribution, incorporating length dependency more accurately:

$P=1-\exp \left[-\left(\frac{L}{L_{0}}\right)^{\alpha} \cdot\left(\frac{\sigma_{f}}{\sigma_{0}}\right)^{m}\right]$

where $P$ is the probability that a segment of fibre of length $L$ will fail when an axial stress $\sigma_{f}$ is applied to it, $L_{0}$ is the reference gauge length, $\sigma_{0}$ is the Weibull strength scale parameter and $m$ is the Weibull shape parameter. The correction exponent $\alpha$ is attributed to correlation of the flaws along the fibre length or to diameter variations within each fibre [35, 38]. The Weibull data set from Beyerlein et al. [27] is used for AS4 carbon fibre, with $\sigma_{0}=4493 \mathrm{MPa}, L_{0}=10 \mathrm{~mm}, m=4.8$ and $\alpha=0.6$. For glass fibres, most available data sets are consistent with the standard Weibull equation with $\alpha=1$. The data set for E-glass is taken from Okabe et al. [39], with $\sigma_{0}=1550 M P a$, $L_{0}=24 \mathrm{~mm}, m=6.34$ and $\alpha=1$. To assign fibre strengths, a random number between 0 and 1 is generated to represent $P$. Equation 1 is then solved to obtain the corresponding strength of the fibre element.

\subsection{Stress concentrations without fibre break interactions}

Identifying and applying the correct stress redistribution during fibre failure is a challenging task in hybrid composites. Therefore, to obtain a tractable procedure, linear stress recovery in the broken fibres is assumed, just as in the Kelly-Tyson model [40]. The ineffective length for these profiles was obtained from FE calculations. It was defined as the relative distance from the break at which $90 \%$ of the nominal fibre stress is recovered. The FE calculations for ineffective length are described in detail in [22, 34].

The assumed stress recovery profiles for both carbon and glass fibres are shown in Fig. 3. The ineffective length yields one point on the stress recovery profile, while the other point on each plot is the origin. The ineffective length in the finite element simulations was found to be $52 \mu \mathrm{m}$ and $26 \mu \mathrm{m}$ for carbon and glass fibre, respectively. The stress recovery in a broken carbon fibre is slower than in a broken glass fibre due to the higher stiffness of the carbon fibre [34]. It has been previously proven that hybridisation has little influence on the ineffective length for each fibre type [22]. The above estimates can thus be used with confidence for hybrid composites. 


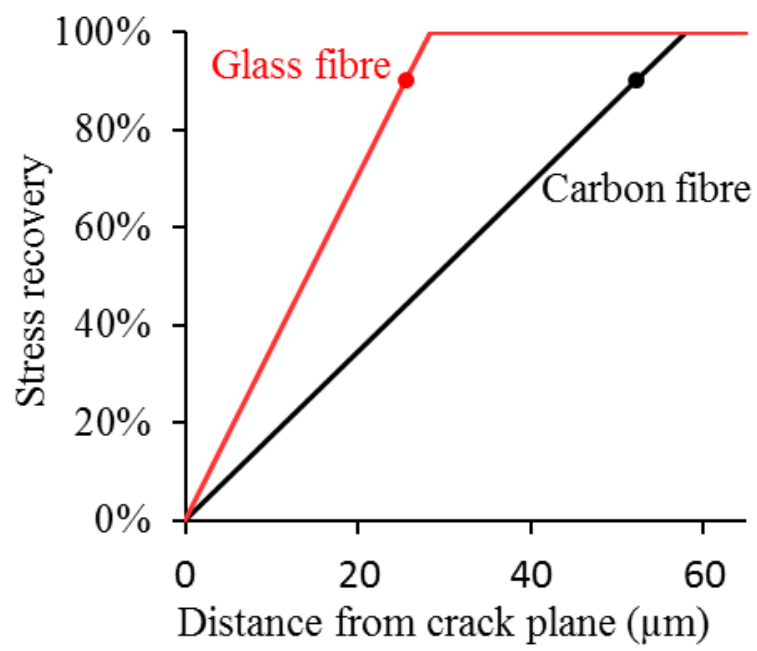

Figure 3: Stress recovery in a single broken fibre as a function of the distance from the crack plane. The red and black dot indicate the two data points coming from the FE calculations for glass and carbon fibre respectively.

"Very local load sharing" between fibres is assumed in this study. In a hexagonal packing, this means that all stress concentrations due to a single fibre break are confined to the 6 nearest neighbouring fibres. In non-hybrid composites, this assumption leads to SCFs in the crack plane being equal to $7 / 6$ for each nearest neighbour around a single fibre break. FE calculations for hexagonal packings have demonstrated that the real SCF is significantly lower than this value [34, 41]. For random fibre packings, however, such high SCFs are actually possible, although only for fibres very near to the broken fibre [34]. Further away from the crack plane, the stress in the broken fibre is increased, as in Fig. 3. Therefore, less load is shed to the surrounding fibres and the SCFs in the intact fibres decrease accordingly.

To determine how the SCFs in hybrid composites are distributed on the carbon and glass fibres, available knowledge on this topic is used. Swolfs et al. [22] demonstrated that the local arrangement of the fibre types in hybrid composites does not affect the SCFs. Glass fibres around a single broken carbon fibre also carried a higher SCF than the carbon fibres around that broken fibre. This was only true if both fibre types are assumed to have the same radius, which is the case here. The ratio of the SCF shed to glass fibres over that shed to carbon fibres was found to be approximately equal to the inverse ratio of their stiffness, $230 \mathrm{GPa} / 70 \mathrm{GPa}$ [22]. This ratio is used throughout the paper. Thus, a single broken carbon fibre is assumed to have nearest neighbour carbon fibres with an SCF equal to 7/6. The nearest neighbour glass fibres then carry an SCF equal to $1+1 / 6 \times 230 / 70=1.548$. This stress redistribution is illustrated in Fig. 4a.

Swolfs et al. [22] did not analyse the stress redistribution around a broken glass fibre. It is assumed that this redistribution is similar to the one around a broken carbon fibre. The single broken glass fibre is assumed to have nearest neighbour glass fibres with an SCF equal to $7 / 6$. The nearest neighbour carbon fibres then carry an SCF equal to $1+$ $1 / 6^{*} 70 / 230=1.051$. Examples of the resulting SCFs for a single fibre break are displayed in Fig. 4b. The SCFs on these 7 fibres, with the broken one having an SCF = 0 , do not add up to 7 as one might expect. This is not required, as the fibre stiffnesses 
are different. Instead, it is the total load on the fibres that must be kept constant before and after the fibre break. The proposed scheme achieves this force equilibrium.

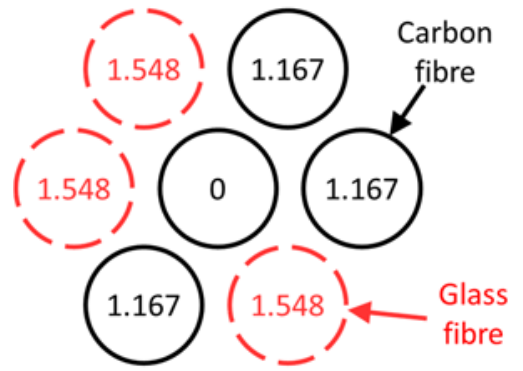

(a)

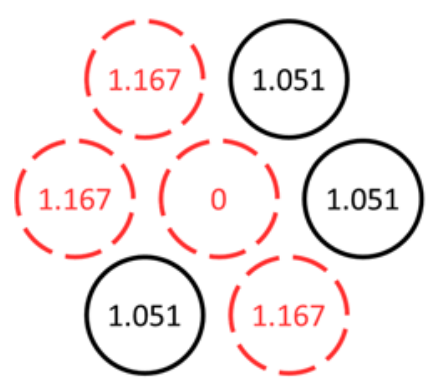

(b)

Figure 4: Illustration of the stress concentration factors (SCFs) in the crack plane according to very local load sharing around (a) a broken carbon fibre, and (b) a broken glass fibre.

\subsection{Stress concentrations with fibre break interactions}

Fig. 4 shows only the SCFs around a single fibre break, while the SCFs around multiple fibre breaks are the crucial ones for predicting final composite failure. Linear superposition of the SCFs can be used, but this is known to underestimate the SCFs around multiple fibre breaks $[42,43]$. Another issue with standard linear superposition is that it does not satisfy force equilibrium, even in non-hybrid composites. Fig. 5a illustrates this issue for a carbon fibre composite with 2 adjacent fibre breaks. Since this composite contains 10 fibres of the same type, the sum of all SCFs should be 10 to avoid loss of load bearing capacity. Standard linear superposition, however, omits the SCF due to one broken fibre on another broken fibre and vice versa. This leads to a sum of 9.667, meaning that force equilibrium is not maintained.

An enhanced linear superposition is developed to resolve this issue. It is proposed to distribute the load shed by breaks in a cluster in proportion to the prediction obtained from standard linear superposition. This distribution causes intact fibres that are nearest neighbours to multiple fibre breaks to have a higher SCF than those adjacent to only one fibre break. This feature is also present in linear superposition. Thus the procedure is to first compute the SCFs by the use of standard linear superposition. The SCFs caused by every individual fibre break are summed up for each fibre element separately. Then, the total load sustained by the broken fibres in the cluster and all of its nearest neighbours are summed up. This sum is compared to the total load prior to the fibre breaks. The SCF in the nearest intact neighbours is adjusted upwards. This adjustment ensures that the total load transmitted by the entire set of fibres agrees with that sustained prior to 
the fibre breaks. The resulting SCFs are shown in Fig. 5b. In this example, the SCF shared by each intact nearest neighbour fibre is adjusted upwards by a factor equal to 6/5. This factor is not applied to the SCF itself, but to the additional SCF, being SCF minus 1 .

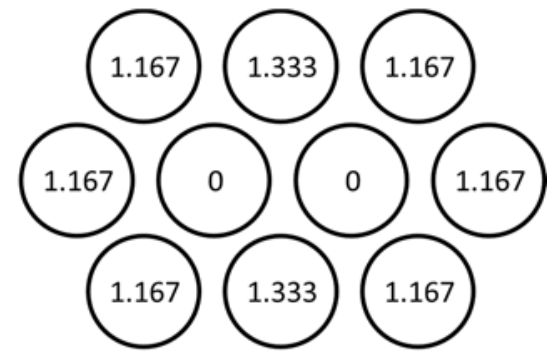

(a)

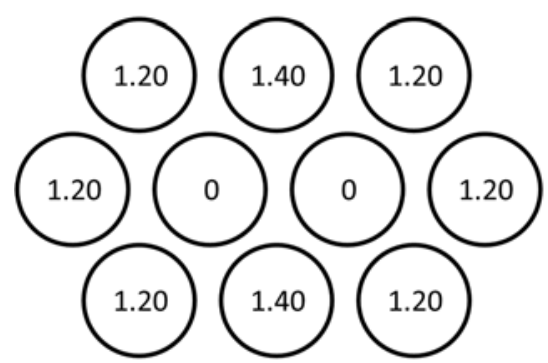

(b)

Figure 5: Stress concentration factors (SCFs) in the crack plane (a) from linear superposition, and (b) enhanced linear superposition in a carbon fibre composite having two fibre breaks in neighbouring fibres where very local load sharing is assumed.

Due to the stress recovery in the broken fibre, it can occur that a fibre fails twice along its length. If this occurs within the stress recovery region, the model assumes that the lowest SCF prevails and no superposition is applied. Similarly, two nearby fibre breaks can potentially cause SCFs within each other's stress recovery region. It was decided not to apply SCFs within the stress recovery region of broken fibres. Instead, these SCFs are taken into account through the enhanced superposition, as explained in the previous paragraph.

The superposition principle leads to preferential break-cluster formation at the edge of the model. Fibres at the edge have fewer nearest neighbours. These fibres hence lead to larger stress concentrations than experienced at break-clusters contained within the interior of the model. To mitigate this feature, a ring of unbreakable fibres with infinite strength is added at the model perimeter. These boundary fibres can carry SCFs, but are not allowed to break and their stress is not used to calculate the composite stress. The boundary fibres are a combination of carbon and glass fibres, depending on the hybrid volume fraction and dispersion type.

\subsection{Break-cluster development}

To understand the hybrid effect in more detail than in Jones and DiBenedetto [44], an algorithm was written to track break-cluster development. Two broken fibre elements are considered to be part of the same break-cluster if (1) they are within $35 \mu \mathrm{m}$ of each 
other in the fibre axial direction, and if (2) they are one of the six nearest neighbours in a hexagonal packing. The first condition is chosen as a compromise between the ineffective lengths for the 2 fibre types. If breaks in nearest neighbour fibres are closer to each other in the axial direction than the ineffective length for those fibres, then those breaks will interact with each other. These breaks are thus considered to form a breakcluster. Both carbon and glass fibres are taken into account for the break-clusters.

\section{Results and discussion}

\subsection{Effect of hybrid volume fraction}

The hybrid volume fraction, defined as the relative volume fraction of glass fibre compared to the overall fibre volume fraction, is varied between $0 \%$ and $100 \%$. The carbon and glass fibres are randomly dispersed, as shown in Fig. 6.
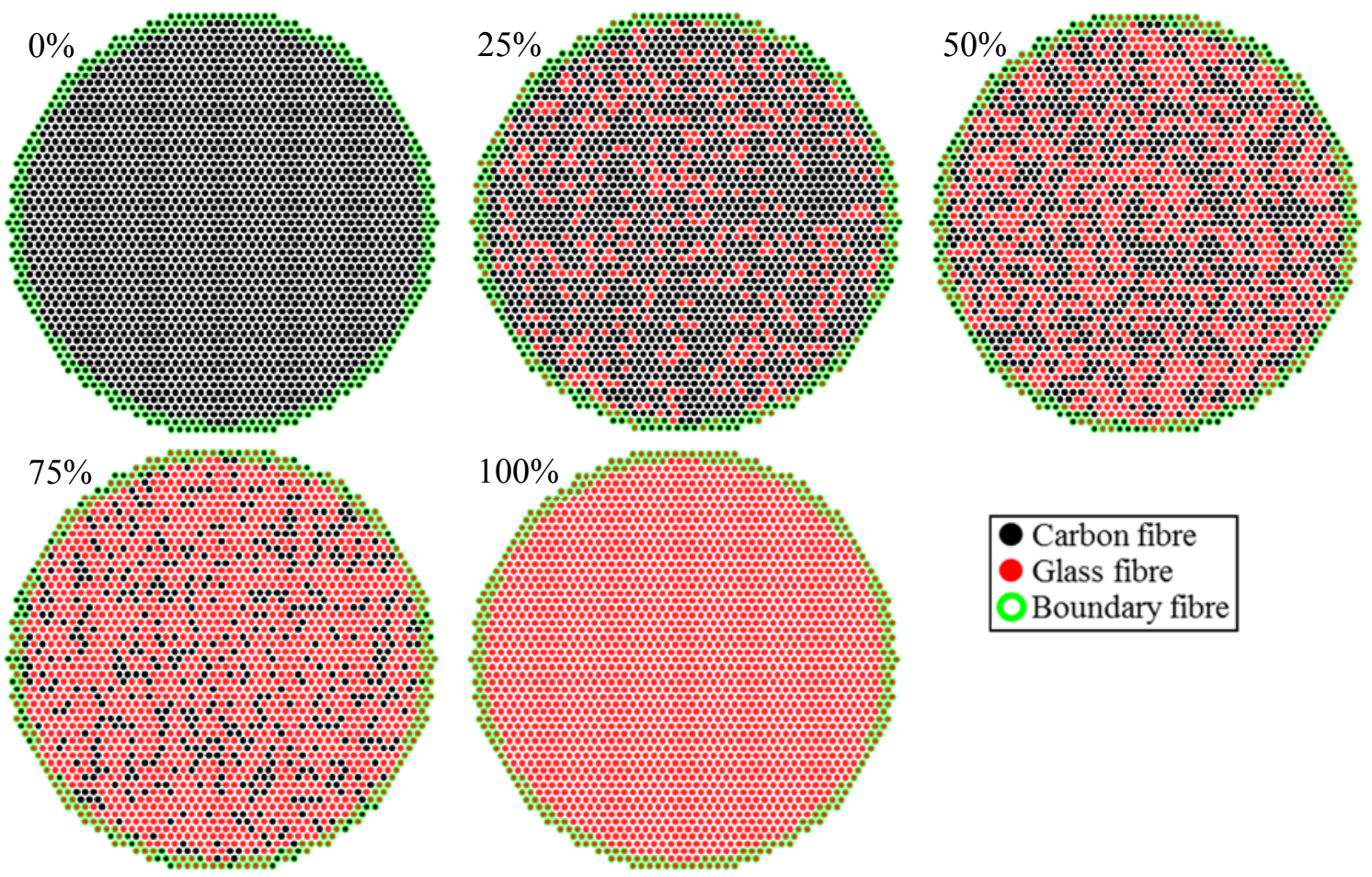

Figure 6: Examples of randomly dispersed packings of carbon/glass at 5 different hybrid volume fractions.

Fig. 7a shows that the stiffness and strength of the hybrid composite decrease with increased hybrid volume fraction, while the failure strain increases noticeably. To investigate this failure strain increase further, the hybrid effect is computed and plotted in Fig. 7b. The hybrid effect is calculated as the relative increase of the failure strain compared to the failure strain of the all-carbon fibre-reinforced composite. This reference failure strain is predicted to be $1.50 \% \pm 0.03 \%$. This agrees well with the $1.55 \%$ failure strain expected for AS4 carbon fibre composites, as mentioned in Hexcel's data sheet [45]. The reference failure strain for glass fibres was $2.82 \% \pm$ $0.06 \%$. While this may be somewhat low for glass fibres, larger glass fibre failure strain did not significantly affect the hybrid effect. 
The hybrid effect gradually increases with increased hybrid volume fractions, see Fig. $7 \mathrm{~b}$. It rises to $32 \%$ at a hybrid volume fraction equal to $90 \%$. The failure of the hybrid composite has to be associated with a critical cluster of carbon fibres that propagates unstably within the same strain increment. If this is not the case, then it cannot be considered a hybrid effect, as defined in section "2.1 General approach". This was indeed confirmed by a thorough analysis of all the modelled configurations.

The trend in Fig. $7 \mathrm{~b}$ corresponds well to the increase expected from experimental data in the review of Kretsis [8]. At a hybrid volume fraction of $100 \%$, the composite has only glass fibres, and its failure strain corresponds to such a material. This should not be interpreted as a hybrid effect, though it is plotted in Fig. $7 \mathrm{~b}$ as such for completeness.

Fig. 7b proves that the failure strain of carbon fibre composites can be increased dramatically if a large fraction of well-dispersed glass fibres is added. Another interesting feature arises when we consider the effect of a small amount of carbon fibre added to a glass fibre composite. When only $10 \%$ of carbon fibres are added to a glass fibre composite, the failure strain is reduced from $2.82 \% \pm 0.06 \%$ to $1.99 \% \pm 0.05 \%$. This is a dramatic reduction for such a small modification of the composite. When the carbon fibres break, they act as crack initiators for the glass fibres. This crack initiation mechanism drastically reduces the failure strain of the hybrid composite.

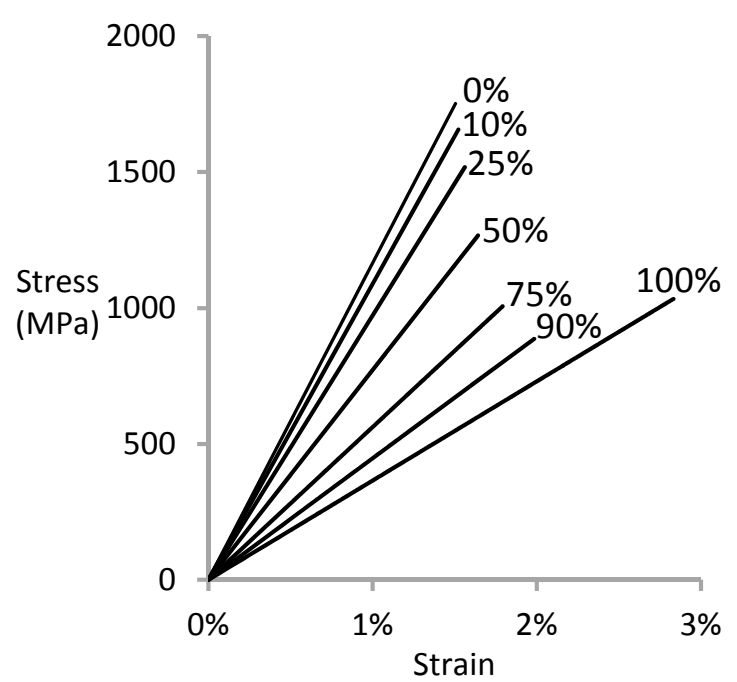

(a)

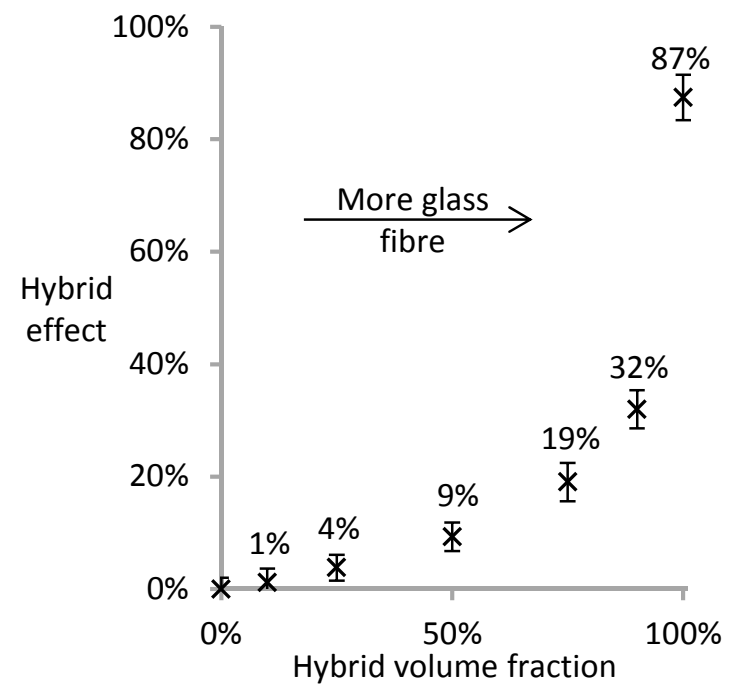

(b)

Figure 7: (a) Stress-strain diagrams, and (b) the hybrid effect (defined as the relative failure strain enhancement of the carbon fibers) for randomly dispersed packings at various hybrid volume fractions for carbon/glass hybrid composites.

The break-cluster information generated by the model is data-rich, making it impossible to present it all. Therefore, the evolution of triplets, which are clusters of 3 breaks, was chosen as a marker for cluster evolution. The conclusions were also checked for other break-cluster sizes, but are not shown here. The definition of a break-cluster can be found in "2.5 Break-cluster development".

The triplet development for various hybrid volume fractions is shown in Fig. 8. It can be seen that the triplet development is most rapid for the all-carbon fibre composite. The 
triplet development is delayed as the hybrid volume fraction is increased and this is especially true for a hybrid volume fraction of $90 \%$. In that case, the probability of finding 3 breaks in carbon fibres next to each other is small. Triplets are only likely to be formed when the strain is high enough for the glass fibres to yield a significant number of breaks.

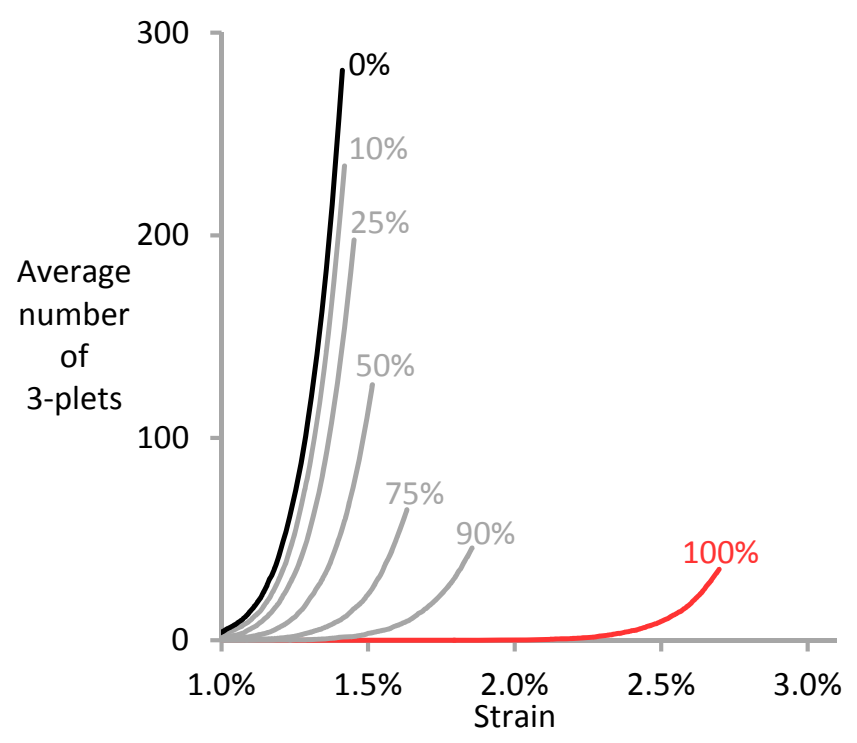

Figure 8: The evolution of triplets (break-clusters of 3 fibres) as a function of strain for randomly dispersed 50/50 hybrid composites.

Finally, the critical cluster size was analysed by identifying the largest break-cluster in the last strain increment before final failure. Fig. 9 indicates an average critical cluster size of 22 broken fibres for all-carbon fibre composites, while it is only 11 for all-glass fibre composites. The SCFs in the crack plane are the same for both non-hybrid composites. This is attributed to the very local load sharing assumptions and SCFs being expressed relative to the nominal fibre stress. Fig. 3 illustrated the longer ineffective length of carbon fibre, which also causes the SCFs to be exerted over longer distances. While this increases the SCFs of more elements than in the case of glass fibres, it also spreads out a possible break-cluster over a longer length. This weakens their interaction and spreads out the SCFs over a longer length. Larger cluster sizes are hence allowed before unstable propagation starts. 


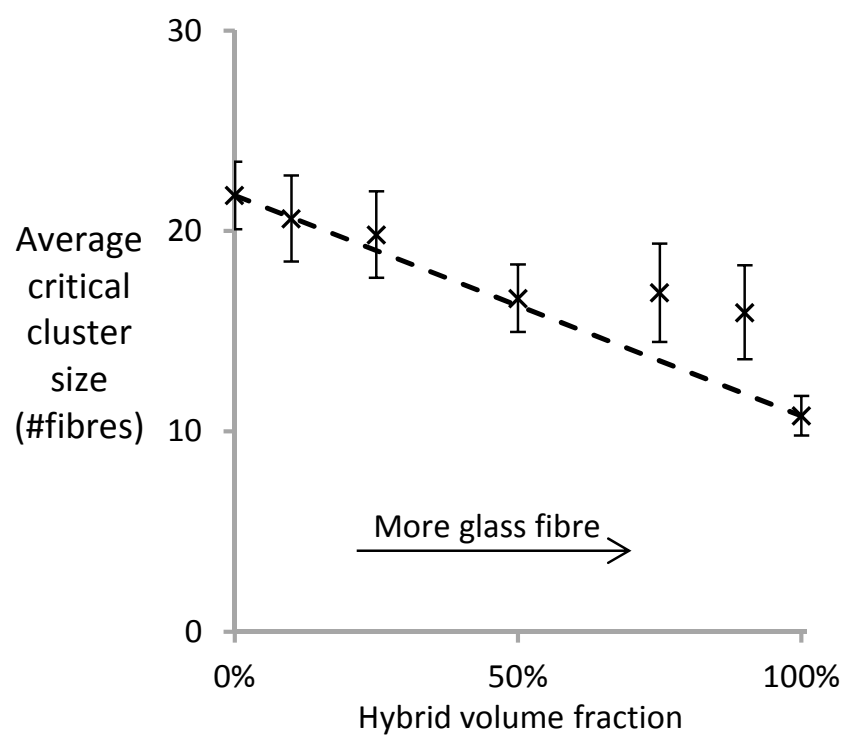

Figure 9: Critical cluster size as a function of the hybrid volume fraction. The dashed line indicates the rule-of-mixtures.

Another influence on the critical cluster size is the difference in the Weibull distributions. To understand the influence of Weibull modulus on the critical cluster size, let us assume a large break-cluster. For fibres with a large strength scatter, the probability that the break-cluster is surrounded by at least a few very strong fibres is larger. These strong fibres make unstable propagation less likely and will require a larger break-cluster before its propagation can occur. Since carbon fibre has the smaller Weibull modulus in this case, it can be expected to have a larger critical cluster size than glass fibres. This trend is confirmed by the analytic expression for critical cluster size in Ibnabdeljalil and Curtin [46].

This reasoning can be extended to hybrid composites with one small alteration. Since stresses in each fibre type are different, the strength scatter argument should be replaced by a reasoning based on strain scatter. The critical cluster size for the hybrid composites lies between values found for the two non-hybrid composites. However, a positive deviation from a linear rule-of-mixtures is found. This positive deviation can delay the development of the critical cluster and hence contribute to a positive hybrid effect. This contribution has not been previously described in literature.

\subsection{Effect of fibre dispersion}

Fibre dispersion is a measure for how well two fibre types in a hybrid composite are mixed. When the hybrid volume fraction is increased, the fibre dispersion is inevitably increased. In this section, the hybrid volume fraction is fixed at $50 \%$ and the influence of the fibre dispersion is analysed. The first dispersion type is bundle-by-bundle, displayed in Fig. 10. The fibre dispersion is labelled by the number of bundles intersected by a horizontal line in the middle of the circular cross-sections shown. The number of carbon fibres in each bundle ranges from 500 for the " 2 bundles"-model down to about 10 for the "16 bundles"-model. Labelling fibre dispersion according to 
the number of fibre bundles in each model would have perhaps been more intuitive. Unfortunately, the circular cross-section of the model leads to incomplete fibre bundles.
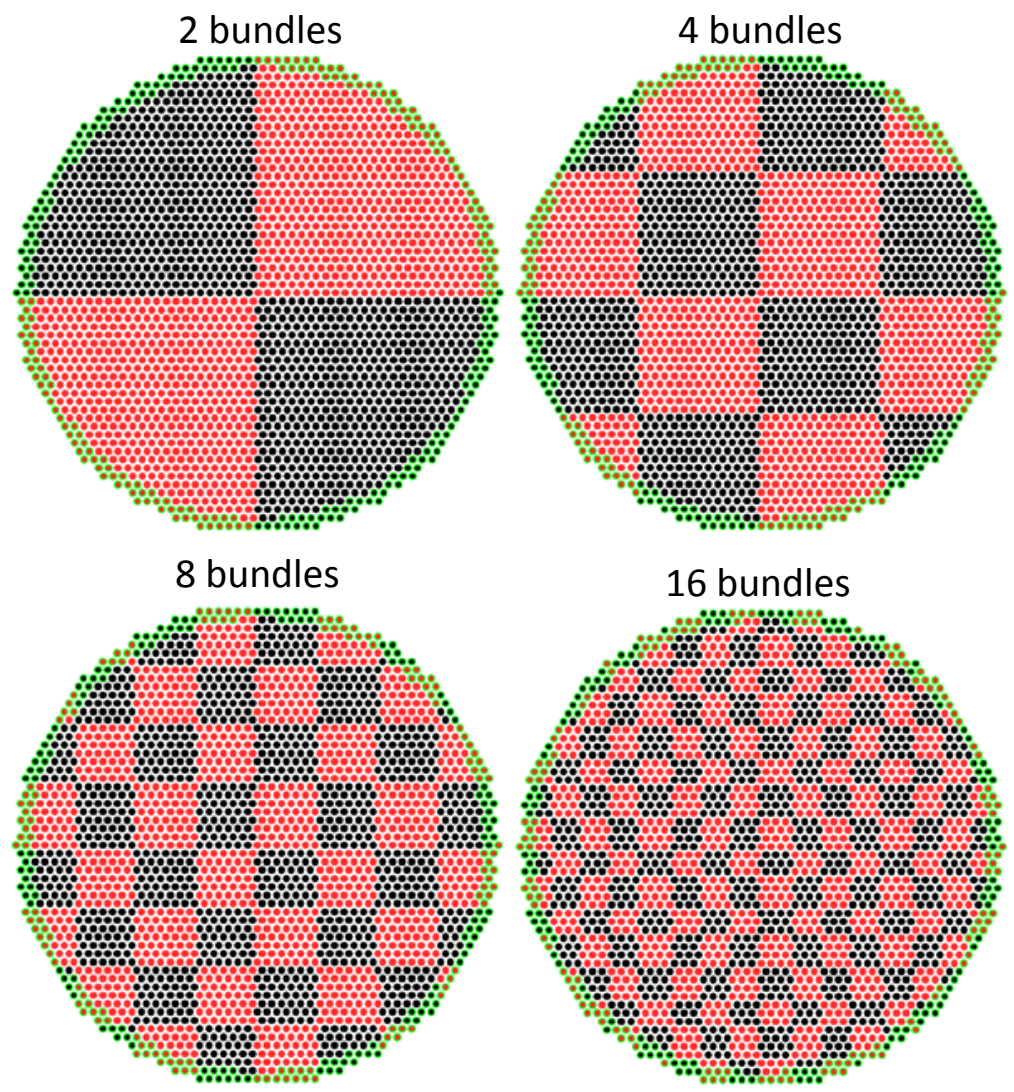

Figure 10: Illustration of bundle-by-bundle dispersion, where black circles are carbon fibres and red denotes glass fibres.

The influence of the bundle size on the hybrid effect and triplet evolution is shown in Fig. 11. For the 2 bundles model, the hybrid effect is only $1.5 \%$, while it increases to $7 \%$ for 16 bundles, as can be seen in Fig. 11a. The latter effect approaches the 9\% hybrid effect found for random dispersion at 50\% hybrid volume fraction. Fig. $11 \mathrm{~b}$ proves that increased dispersion leads to a delay in break-cluster development. A similar delay was also found for other cluster sizes, but is not shown here. 


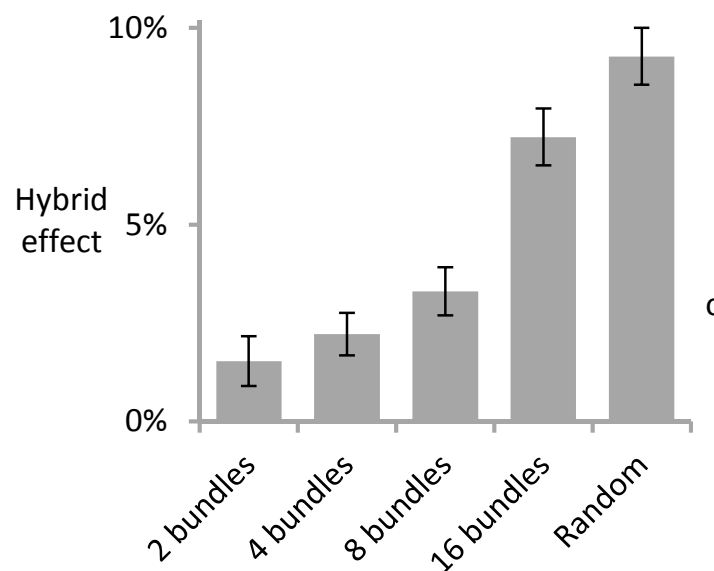

(a)

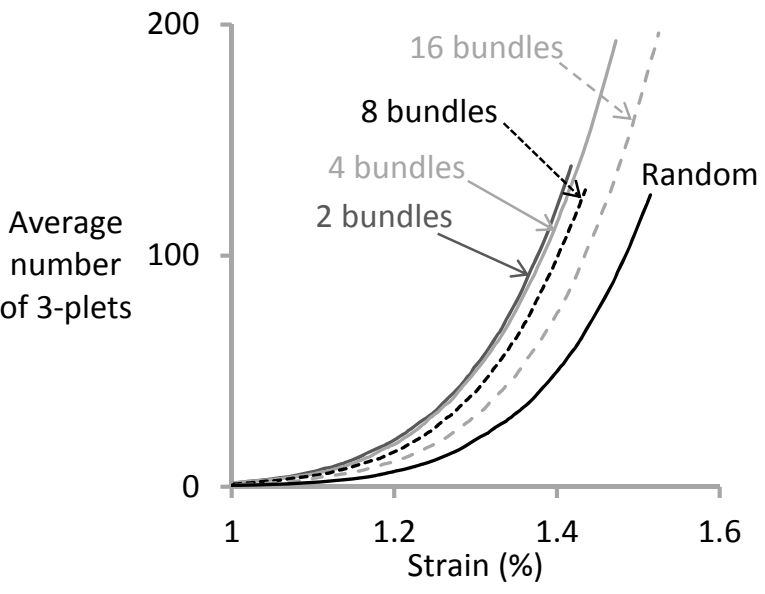

(b)

Figure 11: (a) The hybrid effect for bundle-by-bundle fibre dispersion, and (b) the evolution of triplets (break-clusters of 3 fibres) as a function of strain. The result for random dispersion was added to facilitate comparisons.

The second dispersion type is layer-by-layer, as shown in Fig. 12. The fibre dispersion is labelled according to the number of fibres across the thickness of each layer. The corresponding hybrid effects and sequences of triplet evolution are shown in Fig. 13. Even though these layer-by-layer hybrids seem less dispersed than randomly dispersed hybrids, they are able to reach a higher hybrid effect. For the single fibre layer case, the hybrid effect is $16 \%$, which is significantly higher than the $9 \%$ found for random dispersion.
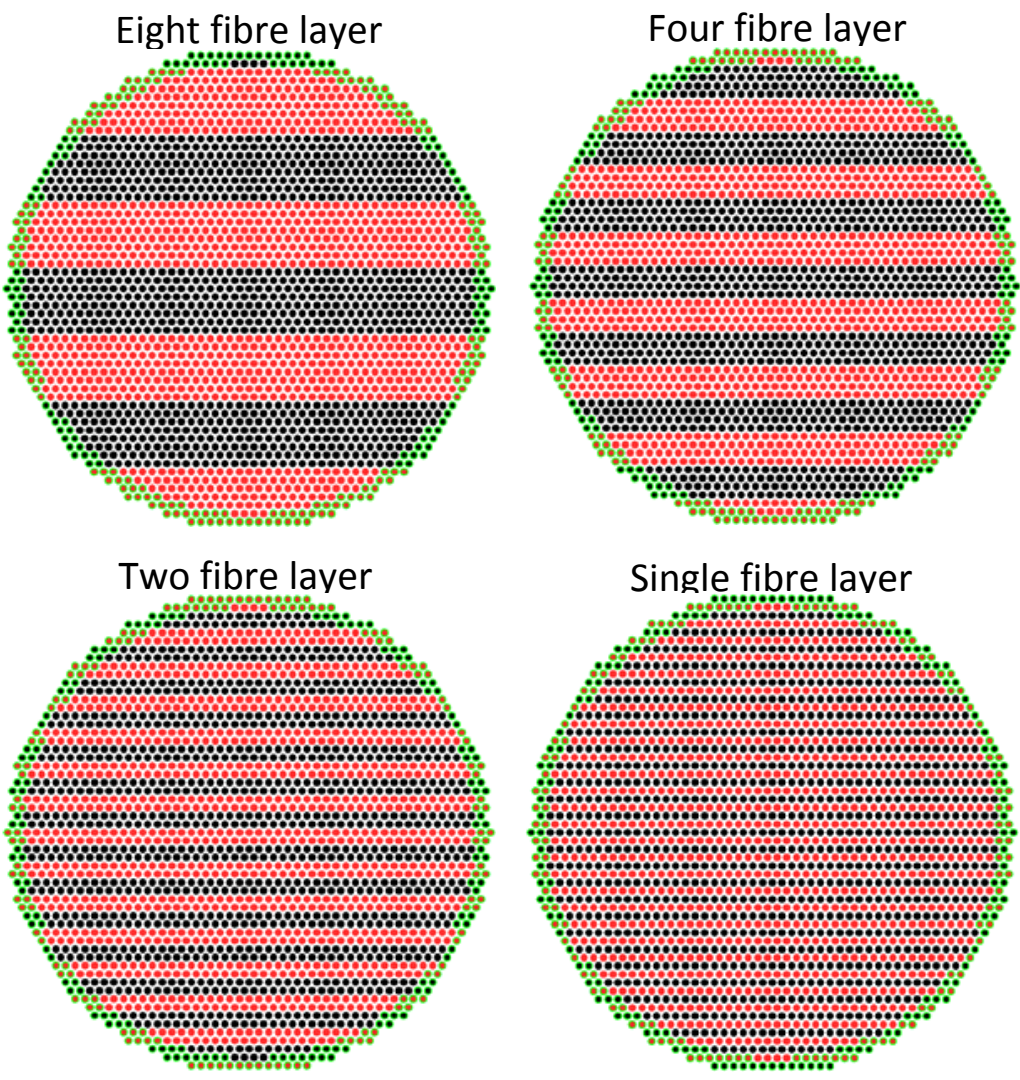
Figure 12: Illustration of layer-by-layer dispersion, where black circles are carbon fibres and red denotes glass fibres.

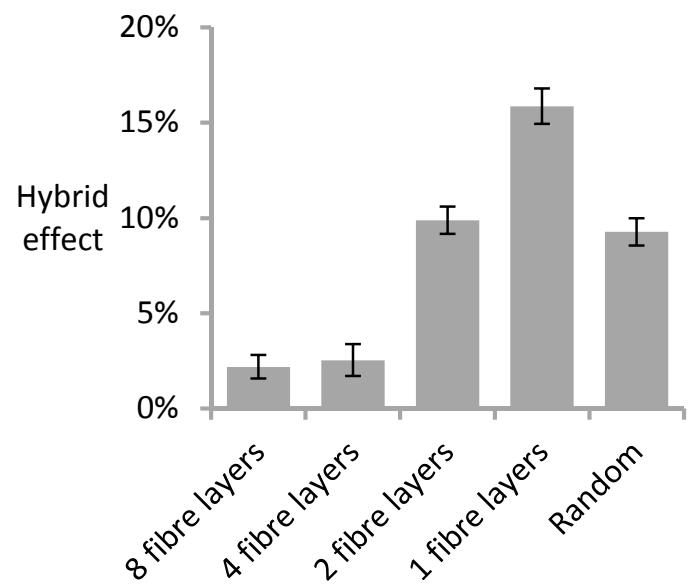

(a)

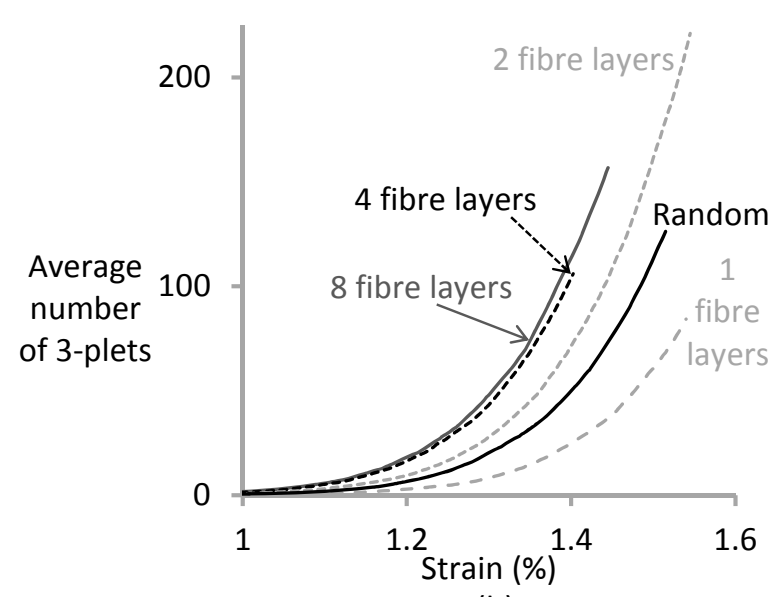

(b)

Figure 13: (a) The hybrid effect for layer-by-layer fibre dispersion, and (b) the evolution of triplets (break-clusters of 3 fibres) as a function of strain. The result for random dispersion is added to facilitate comparisons.

This remarkable effect can be explained based on the sequence of break-cluster development. The development of triplets or break-clusters in general is delayed, because forming break-clusters is more difficult in a single layer of carbon fibres. If one carbon fibre breaks, the nearest neighbours are 2 carbon fibres and 4 glass fibres. The applied strain, combined with the SCF, is then not yet high enough to break glass fibres. Therefore, the break-cluster can only grow in the direction of the layer. If one of the two neighbouring carbon fibres happens to be relatively strong, then there is only one pathway for the break-cluster to grow. In a random packing, on the other hand, the number of pathways for the break-cluster to grow is larger. For a random dispersion with $50 \%$ hybrid volume fraction, the average number of neighbouring carbon fibres is 3. A strong neighbouring fibre is less likely to stop break-cluster development, as there are other pathways for it to grow.

The critical break-cluster sizes for the various fibre dispersions are summarised in Fig. 14. The critical break-cluster size does not vary significantly with dispersion. Most of the observed differences are not statistically significant, despite having 50 simulations for each configuration. The importance of the critical break-cluster size for the hybrid effect is therefore not straightforward. It helps to explain the influence of hybrid volume fraction, but it does not seem to help for the influence of dispersion. It seems that the delay in break-cluster development is the key parameter determining the hybrid effect. 


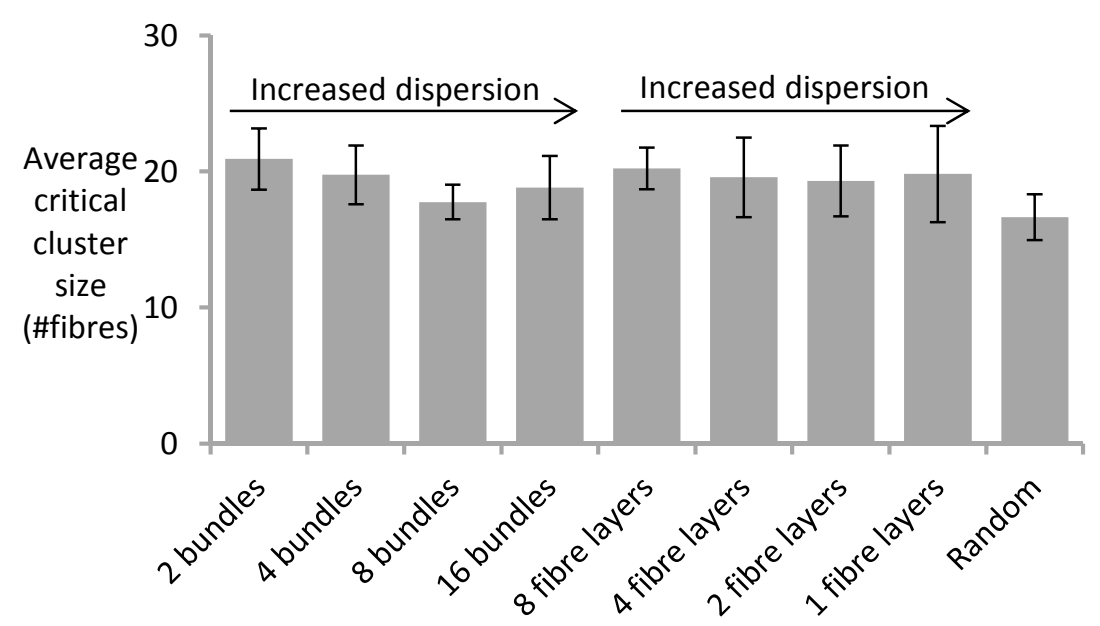

Figure 14: The critical cluster size for various fibre dispersions studied.

In general, these results demonstrate that the dispersion is a vital parameter for the hybrid effect. This was the first study to optimise the hybrid effect by improving dispersion. In contrast with what could have been expected from literature, random dispersion was not the optimal dispersion for maximising the hybrid effect. Layer-bylayer hybrids seem to be more efficient in delaying the failure development, which increases the hybrid effect. From a practical point of view, this would mean that hybrid composites made with thin plies have more potential than comingled hybrids.

\section{Conclusion}

A very local load sharing model for hybrid composites is developed and used to analyse failure development for unidirectional carbon/glass hybrid composites. Addition of glass fibres to a carbon fibre composite increases the failure strain of the composite, an effect known as the hybrid effect. If the relative glass fibre fraction in the hybrid composite is increased, then the development of break-clusters in the carbon fibres is delayed. This delay postpones failure to a higher strain in the composite and thus leads to a more pronounced hybrid effect. This effect was up to $32 \%$ for models with $90 \%$ of glass fibres.

For 50/50 carbon/glass hybrids, the fibre dispersion is proven to have an important influence on the hybrid effect. Random dispersion leads to a hybrid effect of $9 \%$. A slightly smaller hybrid effect was found in bundle-by-bundle dispersion for bundles of only 10 fibres. The highest hybrid effect, however, was found for layer-by-layer hybrids. A hybrid effect of $16 \%$ is found for alternating layers of carbon and glass fibres, each of which are only one fibre thick. By reducing the number of pathways for cluster growth, such layer-by-layer hybrids more efficiently delay the development of break-clusters. The results indicate that thin ply hybrids have more potential for large hybrid effects than comingled hybrids.

Finally, it was shown that the critical cluster size is a function of the hybrid volume fraction. This feature contributes to a positive hybrid effect, as large clusters of fibre breaks are associated with failure of the composite. However, the critical cluster size did 
not depend on the fibre dispersion, meaning that the delay in cluster development is the key parameter controlling the hybrid effect.

This model will be further extended to local load sharing instead of very local load sharing. This requires a more in-depth study of the stress concentrations around broken fibres in hybrid composites, but will yield more accurate results. An experimental validation of this model is currently being performed, and will be reported in an upcoming paper.

\section{Acknowledgments}

The work leading to this publication has received funding from the European Union Seventh Framework Programme (FP7/2007-2013) under the topic NMP-2009-2.5-1, as part of the project HIVOCOMP (Grant Agreement No. 246389). The authors thank the Agency for Innovation by Science and Technology in Flanders (IWT) for a grant given to Y. Swolfs. I. Verpoest holds the Toray Chair at KU Leuven. We acknowledge support from the UCSB Center for Scientific Computing at CNSI, the UCSB MRL: an NSF MRSEC (DMR-1121053) and NSF Grant CNS-0960316.

\section{References}

[1] Hsieh TH, Kinloch AJ, Masania K, Lee JS, Taylor AC, Sprenger S. The toughness of epoxy polymers and fibre composites modified with rubber microparticles and silica nanoparticles. Journal of Materials Science. 2010;45(5):1193-210.

[2] De Greef N, Gorbatikh L, Godara A, Mezzo L, Lomov SV, Verpoest I. The effect of carbon nanotubes on the damage development in carbon fiber/epoxy composites. Carbon. 2011;49(14):4650-64.

[3] Romanov V, Lomov SV, Verpoest I, Gorbatikh L. Can carbon nanotubes grown on fibers fundamentally change stress distribution in a composite? Accepted in Composites Part A: Applied Science and Manufacturing. 2014.

[4] Callens MG, Gorbatikh L, Verpoest I. Ductile steel fibre composites with brittle and ductile matrices. Composites Part A: Applied Science and Manufacturing. 2014;61:23544.

[5] Swolfs Y, Crauwels L, Gorbatikh L, Verpoest I. The influence of weave architecture on the mechanical properties of self-reinforced polypropylene. Composites Part A: Applied Science and Manufacturing. 2013;53:129-36.

[6] Swolfs Y, Crauwels L, Van Breda E, Gorbatikh L, Hine P, Ward I, et al. Tensile behaviour of intralayer hybrid composites of carbon fibre and self-reinforced polypropylene. Composites Part A: Applied Science and Manufacturing. 2014;59:7884.

[7] Hine PJ, Bonner M, Ward IM, Swolfs Y, Verpoest I, Mierzwa A. Hybrid carbon fibre/nylon 12 single polymer composites. Composites Part A: Applied Science and Manufacturing. 2014;65(0):19-26.

[8] Kretsis G. A review of the tensile, compressive, flexural and shear properties of hybrid fibre-reinforced plastics. Composites. 1987;18(1):13-23.

[9] Verpoest I, Lomov S, Swolfs Y, Jacquet P, Michaud V, Manson J-A, et al. Advanced materials enabling high-volume road transport applications of lightweight structural composite parts. Sampe J. 2014;50(3):30-7. 
[10] Hayashi T. On the improvement of mechanical properties of composites by hybrid composition. Proc 8th Intl Reinforced Plastics Conference. 1972:149-52.

[11] You YJ, Park YH, Kim HY, Park JS. Hybrid effect on tensile properties of FRP rods with various material compositions. Composite Structures. 2007;80(1):117-22.

[12] Peijs A, Catsman P, Govaert LE, Lemstra PJ. Hybrid composites based on polyethylene and carbon fibres Part 2: Influence of composition and adhesion level of polyethylene fibers on mechanical properties. Composites. 1990;21(6):513-21.

[13] Manders PW, Bader MG. The strength of hybrid glass/carbon fibre composites Part 1 Failure strain enhancement and failure mode. Journal of Materials Science. 1981;16(8):2233-45.

[14] Taketa I, Ustarroz J, Gorbatikh L, Lomov SV, Verpoest I. Interply hybrid composites with carbon fiber reinforced polypropylene and self-reinforced polypropylene. Composites Part A: Applied Science and Manufacturing. 2010;41(8):927-32.

[15] Zweben C. Tensile strength of hybrid composites. Journal of Materials Science. 1977;12(7):1325-37.

[16] Ren PG, Zhang ZP, Xie L, Ren F, Jin YL, Di YY, et al. Hybrid Effect on Mechanical Properties of M40-T300 Carbon Fiber Reinforced Bisphenol A Dicyanate Ester Composites. Polymer Composites. 2010;31(12):2129-37.

[17] Pitkethly MJ, Bader MG. Failure modes of hybrid composites consisting of carbon fibre bundles dispersed in a glass fibre epoxy resin matrix. Journal of Physics DApplied Physics. 1987;20(3):315-22.

[18] Xing J, Hsiao GC, Chou TW. A dynamic explanation of the hybrid effect. Journal of Composite Materials. 1981;15(SEP):443-61.

[19] Jalalvand M, Czél G, Wisnom MR. Numerical modelling of the damage modes in UD thin carbon/glass hybrid laminates. Composites Science and Technology. 2014;94:39-47.

[20] Czél G, Wisnom MR. Demonstration of pseudo-ductility in high performance glass/epoxy composites by hybridisation with thin-ply carbon prepreg. Composites Part A: Applied Science and Manufacturing. 2013;52:23-30.

[21] Fukuda H. An advanced theory of the strength of hybrid composites. Journal of Materials Science. 1984;19(3):974-82.

[22] Swolfs Y, Gorbatikh L, Verpoest I. Stress concentrations in hybrid unidirectional fibre-reinforced composites with random fibre packings. Composites Science and Technology. 2013;85:10-6.

[23] Phillips LN. The hybrid effect - does it exist? Composites. 1976;7(1):7-8.

[24] Manders PW, Bader MG. The strength of hybrid glass/carbon fibre composites Part 2 A statistical model. Journal of Materials Science. 1981;16(8):2246-56.

[25] Harlow DG. Statistical properties of hybrid composites. I. Recursion analysis. Proceedings of the Royal Society of London Series A: Mathematical Physical and Engineering Sciences. 1983;389(1796):67-100.

[26] Curtin WA. Dimensionality and size effects on the strength of fiber-reinforced composites. Composites Science and Technology. 2000;60(4):543-51.

[27] Beyerlein IJ, Phoenix SL. Statistics for the strength and size effects of microcomposites with four carbon fibers in epoxy resin. Composites Science and Technology. 1996;56(1):75-92. 
[28] Wisnom MR, Khan B, Hallett SR. Size effects in unnotched tensile strength of unidirectional and quasi-isotropic carbon/epoxy composites. Composite Structures. 2008;84(1):21-8.

[29] Fukuda H, Chou TW. Stress concentrations in a hybrid composite sheet. Journal of Applied Mechanics - Transactions of the ASME. 1983;50(4A):845-8.

[30] Mishnaevsky Jr L, Dai G. Hybrid carbon/glass fiber composites: Micromechanical analysis of structure-damage resistance relationships. Computational Materials Science. 2014;81:630-40.

[31] Dai G, Mishnaevsky Jr L. Fatigue of hybrid glass/carbon composites: 3D computational studies. Composites Science and Technology. 2014;94:71-9.

[32] Rosen BW. Tensile failure of fibrous composites. AIAA Journal. 1964;2(11):198591.

[33] Swolfs Y, Gorbatikh L, Verpoest I. Fibre hybridisation in polymer composites: a review. Composites Part A: Applied Science and Manufacturing. 2014;67:181-200.

[34] Swolfs Y, Gorbatikh L, Romanov V, Orlova S, Lomov SV, Verpoest I. Stress concentrations in an impregnated fibre bundle with random fibre packing. Composites Science and Technology. 2013;74(0):113-20.

[35] Watson AS, Smith RL. An examination of statistical theories for fibrous materials in the light of experimental data. Journal of Materials Science. 1985;20(9):3260-70.

[36] Gutans J, Tamuzs V. Strength probability of unidirectional hybrid composites. Theoretical and Applied Fracture Mechanics. 1987;7(3):193-200.

[37] Okabe T, Takeda N. Size effect on tensile strength of unidirectional CFRP composites - experiment and simulation. Composites Science and Technology. 2002;62(15):2053-64.

[38] Curtin WA. Tensile strength of fiber-reinforced composites: III. Beyond the traditional Weibull model for fiber strengths. Journal of Composite Materials. 2000;34(15):1301-32.

[39] Okabe T, Takeda N, Kamoshida Y, Shimizu M, Curtin WA. A 3D shear-lag model considering micro-damage and statistical strength prediction of unidirectional fiberreinforced composites. Composites Science and Technology. 2001;61(12):1773-87.

[40] Kelly A, Tyson WR. Tensile properties of fibre-reinforced metals - copper/tungsten and copper/molybdenum. Journal of the Mechanics and Physics of Solids. 1965;13(6):329-50.

[41] Nedele MR, Wisnom MR. Stress-Concentration Factors around a Broken Fiber in a Unidirectional Carbon-Fiber-Reinforced Epoxy. Composites. 1994;25(7):549-57.

[42] Swolfs Y, Gorbatikh L, Verpoest I. Stress concentrations around multiple fibre breakages: the validity of linear superposition. 4th ECCOMAS Thematic Conference on Mechanical Response of Composites. Ponta Delgada, Portugal2013.

[43] Behzadi S, Curtis PT, Jones FR. Improving the prediction of tensile failure in unidirectional fibre composites by introducing matrix shear yielding. Composites Science and Technology. 2009;69(14):2421-7.

[44] Jones KD, Dibenedetto AT. Fiber fracture in hybrid composite systems. Composites Science and Technology. 1994;51(1):53-62.

[45] AS4 carbon fibre data sheet, www.hexcel.com, 2014.

[46] Ibnabdeljalil M, Curtin WA. Strength and reliability of fiber-reinforced composites: Localized load-sharing and associated size effects. International Journal of Solids and Structures. 1997;34(21):2649-68. 